Research Article

\title{
Pathway-Wide Genetic Risks in Chlamydial Infections Overlap between Tissue Tropisms: A Genome-Wide Association Scan
}

\author{
Chrissy H. Roberts $\mathbb{D}^{1},{ }^{1}$ Sander Ouburg $\mathbb{D},{ }^{2}$ Mark D. Preston, ${ }^{3}$ Henry J. C. de Vries, ${ }^{4,5,6}$ \\ Martin J. Holland, ${ }^{1}$ and Servaas A. Morré $\left(\mathbb{D}^{2,7}\right.$ \\ ${ }^{1}$ Department of Clinical Research, London School of Hygiene and Tropical Medicine, London, UK \\ ${ }^{2}$ Institute for Public Health Genomics, Department of Genetics and Cell Biology, School for Oncology and Developmental Biology \\ (GROW), Faculty of Health, Medicine and Life Sciences, Maastricht University, 6200 MD Maastricht, Netherlands \\ ${ }^{3}$ National Institute for Biological Standards and Controls, Potters Bar, UK \\ ${ }^{4}$ STI Outpatient Clinic, Department of Infectious Diseases, Public Health Service of Amsterdam, Amsterdam, Netherlands \\ ${ }^{5}$ Amsterdam Infection and Immunity Institute (AIßII), Academic Medical Centre, University of Amsterdam, \\ Amsterdam, Netherlands \\ ${ }^{6}$ Department of Dermatology, Academic Medical Center, University of Amsterdam, Amsterdam, Netherlands \\ ${ }^{7}$ Laboratory of Immunogenetics, Department of Medical Microbiology and Infection Control, VU University Medical Center, \\ 1081 HV Amsterdam, Netherlands
}

Correspondence should be addressed to Chrissy H. Roberts; chrissyhroberts@yahoo.co.uk

Received 20 October 2017; Revised 11 January 2018; Accepted 8 February 2018; Published 3 June 2018

Academic Editor: Vinicius M. Fava

Copyright ( 92018 Chrissy H. Roberts et al. This is an open access article distributed under the Creative Commons Attribution License, which permits unrestricted use, distribution, and reproduction in any medium, provided the original work is properly cited.

\begin{abstract}
Chlamydia trachomatis is the most commonly diagnosed bacterial sexually transmitted infection and can lead to tubal factor infertility, a disease characterised by fibrosis of the fallopian tubes. Genetic polymorphisms in molecular pathways involving $G$ protein-coupled receptor signalling, the Akt/PI3K cascade, the mitotic cell cycle, and immune response have been identified in association with the development of trachomatous scarring, an ocular form of chlamydia-related fibrotic pathology. In this case-control study, we performed genome-wide association and pathways-based analysis in a sample of 71 Dutch women who attended an STI clinic who were seropositive for Chlamydia trachomatis antibodies and 169 high-risk Dutch women who sought similar health services but who were seronegative. We identified two regions of within-gene SNP association with Chlamydia trachomatis serological response and found that GPCR signalling and cell cycle pathways were also associated with the trait. These pathway-level associations appear to be common to immunological sequelae of chlamydial infections in both ocular and urogenital tropisms. These pathways may be central mediators of human refractoriness to chlamydial diseases.
\end{abstract}

\section{Introduction}

Chlamydia trachomatis $(\mathrm{Ct})$ is diagnosed as the cause of around 106 million sexually transmitted infections (STIs) per annum [1] and has a significant impact on global health.

The primary pathology associated with Ct infection is an aberrant host immune response [2] which can lead to the progressive formation of scar tissues at and near the site of infection [3-5]. In the context of STIs, this process can lead to infection-related tubal factor infertility (TFI) and ectopic pregnancy [6]. Ct is also the leading infectious cause of blindness [7], and this form of disease (known as trachoma) is characterised by scar formation on the inner conjunctival surface of the upper eyelid, leading to lid deformation, corneal damage, and visual impairment. Trachoma has historically been used as a platform for the study of chlamydial diseases and their pathology. This is largely due to the relative ease with which the conjunctiva can be studied compared to 
the fallopian tubes but also because of the high prevalence of trachomatous scarring in sub-Saharan Africa [8, 9] compared to Ct pathology [10] in Europe and elsewhere.

Whilst serological evidence of historical Ct STIs can be detected in around a quarter of Northern European women without current Ct infections $[10,11]$, it can be very difficult to ascertain individuals for study who have laparoscopically confirmed cases of TFI with tubal scarring and concomitant seropositivity for historical Ct infections (Ct/TFI). In Europe, the Ct/TFI prevalence in women is likely to be very low, and in the Netherlands, this was estimated at around 0.08\% [10]. This makes the recruitment of participants to research studies focussed on urogenital chlamydial disease difficult. The collection of statistically powered case-control samples is extremely challenging, but it is possible to leverage robust genetic association data from small samples by deploying statistical tests that not only collapse the highly dimensional genetic data to raise power [12] but also iterate over alternate scenarios to eliminate the burden of multiple testing [13]. We have used a pathways-based association test that not only corrects for the length of genes and pathways but also utilises a process of permutation in which the genotypic associations of the observed traits were compared to a large number of simulated trait data sets. This process ensures that any pathways that were ultimately linked to the trait are not significant simply because of structure within the data, or because of limitations of the method, or because of the specific list of pathways that was chosen.

In our recent discovery phase genome-wide association study (GWAS) [14], we found that a number of genes and molecular pathways were associated with TS. That study applied both a classical single-nucleotide polymorphism (SNP) association and systems-based analysis [15-17] that focussed on the cumulative effects of multiple SNPs in the context of well-defined and curated molecular pathways of protein-protein interactions [18]. The trachoma GWAS identified genome-wide significant pathways that were associated with the protection and predisposition to TS. These results indicated a role for genes controlling the immune response, the mitotic cell cycle, and numerous cell surface receptors and downstream signalling factors of the $G$ protein-coupled receptor family. The inference was that the key factors which protect the host from scarring were primarily innate barriers to initial infection and establishment of the intracellular niche, rather than direct protection from the scarring process itself. This interpretation raised a question about whether genetic protection might be so extensive in some people that they are innately refractory to becoming infected. In these people, we hypothesise that infection might never progress to the point that stimulates a lasting adaptive serological or cellular immune response.

The aim of this work was to assess the extent to which sexually transmitted $\mathrm{Ct}$ infections might share a common pathway of genetic predisposition with trachoma. By focussing on a trait of seropositivity for anti-Ct antibodies in high-risk, presumably exposed Dutch women attending STI clinics, we investigated if pathway-wide polymorphisms in the host are associated to primary events of infection. This work takes the form of a GWAS in a small case-control sample in which we tested the association of 1345 multigene pathways, 590,811 directly genotyped SNPs, and 8,852,410 imputed SNPs with serological status in 240 women attending a sexual health clinic in the Netherlands.

\section{Methods}

2.1. Ethics Statement. This study used archival material and linked data from clinical tests that had been irreversibly anonymised. The Medical Research Involving Human Subjects Act (WMO, Dutch Law) states that official approval of the study by the Medical Ethical Committee is not required for research using the anonymous human material collected for this study (MEC Letter reference: number 10.17.0046). All subjects gave written informed consent for their specimens and data to be used in this way. All antecedent clinical studies were conducted in accordance with the tenets of the Declaration of Helsinki.

2.2. Study Population, Sampling, and Ascertainment. 244 Dutch Caucasian women were selected from a larger study consisting of Dutch Caucasian women visiting an outpatient sexual health clinic in Amsterdam, Netherlands, between July 2001 and December 2004. The women used a self-completion questionnaire to describe their symptoms at presentation, which varied from increased vaginal discharge, having bloody discharge during and/or after coitus, recent abdominal pain (not gastrointestinal or menses related), and/or dysuria.

2.3. Genotyping and Quality Control. All specimens $(n=244)$ were genotyped at 713,599 SNPs using the Infinium OmniExpress-24 v1.2 Kit (Illumina). Basecalling was performed using Illuminus [19]. SNPs were retained in the analysis if (a) the call rate was $\geq 0.99$, (b) the minor allele frequency was $\geq 0.01$, and (c) Hardy-Weinberg equilibrium $p$ value $>5 \times 10^{-8}$. 590,811 directly genotyped SNPs were retained after QC.

PLINK and $\mathrm{R}$ were used to test for between-individual proportional IBS variance and to perform kinship estimation by identity by state/allele sharing estimation. Individuals were removed if they were identified as being outliers because they had (a) $>10 \%$ missing genotype data, (b) identity by descent (IBD) sharing with another individual of two alleles at all loci, (c) identity by state with the fifth nearest neighbour with $Z<-4$ compared to the mean IBS of all possible pairs, or (d) unresolved gender mismatch between sex chromosome genotypes and clinical record. After these QC steps, 240 specimens were retained as one failed the pairwise IBD test and three failed the fifth nearest neighbour-based outlier analysis.

2.4. Statistical Power. The R package "gap" [20] was used to estimate the power of the study to detect genome-wide significant $\left(<1 \times 10^{-8}\right)$ associations of individual SNPs. At this level of significance, a study of 71 cases and 169 controls has $80 \%$ power to detect allele frequency odds ratios (OR) of 22.89 , for minor allele frequency (MAF) in the control group of 0.1 . The small sample size of this study meant that we could not differentiate between null results and false negatives. The false positive rate of SNP-based genome-wide association 
tests was meanwhile controlled using the Bonferroni process. The false discovery rate of pathway analysis was controlled using a robust permutation process, which allows multiple testing at no increased cost to power $[12,13]$.

2.5. Imputation. Imputation was carried out as described previously $[21,22]$ and included prephasing with SHAPEIT $[23,24]$ (informed by HapMap Phase II, build 37) and postimputation filtering for high-quality imputed SNPs with IMPUTE2 info score $>0.8$ and/or MAF $>0.01[14,25]$. Imputation was performed with IMPUTE2 [21, 22] using the worldwide 1000 Genomes phase I data set of 1092 reference specimens [26]. 20,514,944 SNPs were imputed, and after filtering, 9,443,221 SNPs were retained for association testing analysis.

2.6. Tests of Association. We modelled the clinical status (seropositive/seronegative) according to age using logistic regression. Residuals of the regression were taken forward as the serological status trait in the association test. Tests of association of SNPs with age-corrected phenotypes were performed using EMMAX [27]. All tests of association were performed using a pairwise matrix of IBS kinship estimates which was generated from the SNP data. SNPnexus (http://snp-nexus. org) was used to annotate the SNPs with $P<1 * 10^{-6}$.

2.7. Pathway Analyses. The R package SNPath (https://github. com/lschen-stat/SNPath) was used to run the Assignment List GO AnnotaTOR (ALIGATOR) algorithm [12] on 1345 Reactome pathways [18]. In order to make the results directly comparable to those of the previous study [14], we chose to use the same pathway list that was used in that analysis (http://www.reactome.org/, accessed April 2013). The $p$ values from EMMAX tests of association were sampled to remove SNPs that were $>20 \mathrm{~kb}$ away from any gene in a list of $\sim 17,000$ genes that were consistently crossreferenced between Entrez and Ensembl and for which a HUGO gene name had been assigned https://github.com/ lschen-stat/SNPath. ALIGATOR requires the user to specify a nominally significant threshold value, and particularly when the sample size is low, this choice can significantly affect the membership of the list of significant pathways. More stringent values for this threshold (i.e., 0.0001) will focus on SNPs most likely to be true positives (at the level of SNP) whilst more relaxed (i.e., 0.01) thresholds will capture more information from genes of small individual effect and may give a better indication of pathway involvement in diseases [12]. We chose to investigate the way in which threshold affected membership of the results table by running ALIGATOR at three thresholds of $P_{\text {EMMAX }}$ $(0.0001,0.001$, and 0.01$)$ and combining the results. We used 5000 permutations of the $P_{\text {EMMAX }}$ values at each threshold. A list of all genes that appeared in any significant pathway at any threshold was generated, and this list was functionally annotated using DAVID Bioinformatics Resources 6.7 [28] to identify enriched Reactome pathways and GO terms, whilst accounting for the significant gene content overlap in specific pathways.
2.8. Serological and Infection Status Determination. Peripheral venous blood was collected for the analysis of IgG antibodies against $C$. trachomatis (CT) using Chlamydia trachomatis-IgG-ELISA plus medac (Medac Diagnostika $\mathrm{mbH}$, Hamburg, Germany). This test uses a synthetic peptide of a variable domain from an immune-dominant region of the major outer membrane protein (MOMP) and has been shown to perform with high specificity ( 0.95-0.97) when evaluated against both Pgp3 and MIF [29]. A Ct IgG titre of $\geq 1: 50$ was considered positive. Samples with values within $\pm 10 \%$ of the $1: 50$ cut-off value were repeated and considered positive when the result was positive or still within the $\pm 10 \%$ range of the cut-off $[30,31]$. The distribution of $\operatorname{IgG}$ titres in the positive population was as follows: titre $(\mathrm{T})=1: 50$ $[n=9], T=1: 100[n=19], T=1: 200[n=24], T=1: 400$ $[n=9], T=1: 800[n=7]$, and $T=1: 1600[n=3]$. In the negative population, all specimens had a titre of $T=1: 1$ $[n=169]$. Tests for infection were performed on endocervical swabs using the COBAS AMPLICOR CT/NG Test (Roche, Basel, Switzerland).

\section{Results}

There were 71 seropositive and 169 seronegative individuals $(n=240)$ in the analysis. All 71 seropositive cases were additionally infection-positive, whilst all 169 seronegative cases were infection-negative. Cases were significantly younger than controls in this sample $(p=0.007)$. The median age of cases was 23 (min 16, 1st quartile 19.5, mean 22.6 , 3rd quartile 24.5, and $\max 30$ ). The median age of controls was 24 (min 15, 1st quartile 21, mean 23.8, 3rd quartile 27, and max 32). The genome-wide inflation statistic was $=1.04$ before correcting for age and $=1.02$ after correction (Supplementary Figure 1).

In the genome-wide association study (GWAS), 46 singlenucleotide polymorphisms (SNPs) with $P_{\text {EMMAX }}<1 E-6$ were identified (see Supplementary Table 1). These were located in five regions ( $\mathrm{A}-\mathrm{E}$, see Table 1). Although none of the individual SNPs reached genome-wide significance $(1 E-8)$, regions B (Figure $1(\mathrm{~b})$ ) and C (Figure $1(\mathrm{c})$ ) contained 33 and 12 SNPs, respectively.

The 33 SNPs in region B lay within the nonproteincoding NPSR Antisense RNA1 (NPSR1-AS1) and immediately upstream of the $G$ protein-coupled receptor coding neuropeptide S receptor 1 (NPSR1) (Figure 1(b)). The 12 SNPs in region $\mathrm{C}$ lie in the protein kinase, CGMP-dependent, type I (PRKG1) (Figure 1(c)), a mediator of the nitric oxide/ cGMP signalling pathway, which has roles in immune function and in GPCR signalling.

Sixty-six differently named Reactome pathways were identified as significantly enriched $\left(p_{\text {perm }}<0.05\right)$ in chlamydial seropositivity. These pathways (Supplementary Table 2) are the combination of results from three ALIGATOR [12] pathway analyses with SNP cut-off thresholds of $0.01,0.001$, and 0.0001 . The number of SNPs reaching each of the cut-off values were 93,106 $(p<$ $0.01), 9998(p<0.001)$, and $1168(p<0.0001)$. The numbers of SNPs mapping to genes at each threshold were 


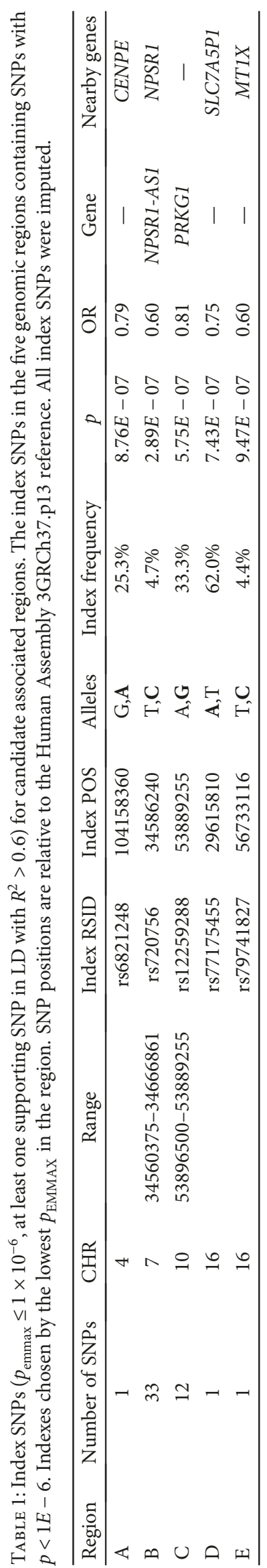



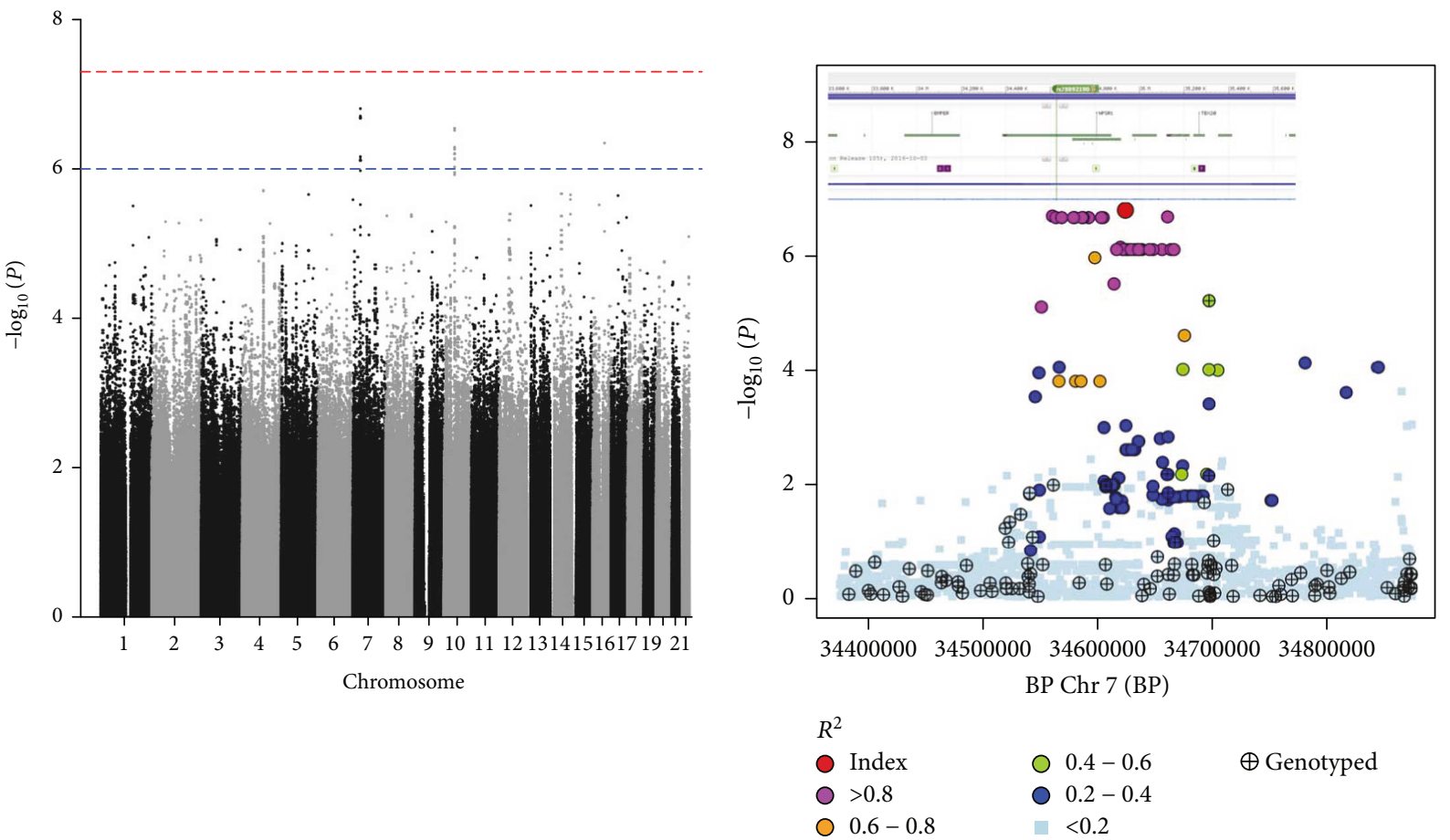

(a)

(b)

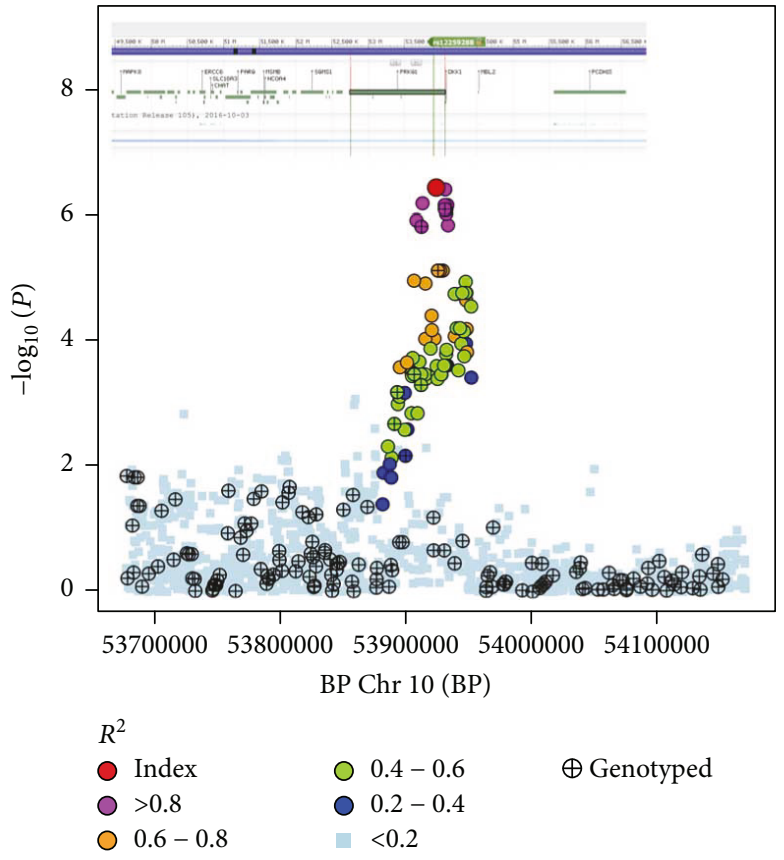

(c)

FIgURE 1: Results of GWAS analysis for Chlamydia trachomatis seropositivity using EMMAX. (a) Manhattan plot showing index SNPs with $p_{\text {EMMAX }}<1 \times 10-6$. There were two regions of within-gene SNP association with Chlamydia trachomatis serological response. (b) The Chr 7: 34560375-34666861 region, within the nonprotein-coding NPSR1 antisense RNA 1 (NPSR1-AS1) and immediately upstream of the G protein-coupled receptor coding neuropeptide S receptor 1 (NPSR1). (c) The Chr 10: 53873323-56733116 region, within the gene encoding protein kinase, CGMP-dependent, type I (PRKG1), a mediator of the nitric oxide/cGMP signalling pathway, which has roles in immune function and GPCR signalling.

64,397 $(p<0.01), 6.274(p<0.001)$, and $451(p<0.0001)$. The number of significant pathways at each cut-off were 17 $(p<0.01), 20(p<0.001)$, and $33(p<0.0001)$. In total, these pathways contained 2556 unique genes. Clustering the
Reactome pathways by genic intersection demonstrated a large gene overlap (Figure 2).

The Reactome "Signalling by GPCR" and "Cell Cycle, Mitotic" pathways contained 563 and 299 genes out of 
Height

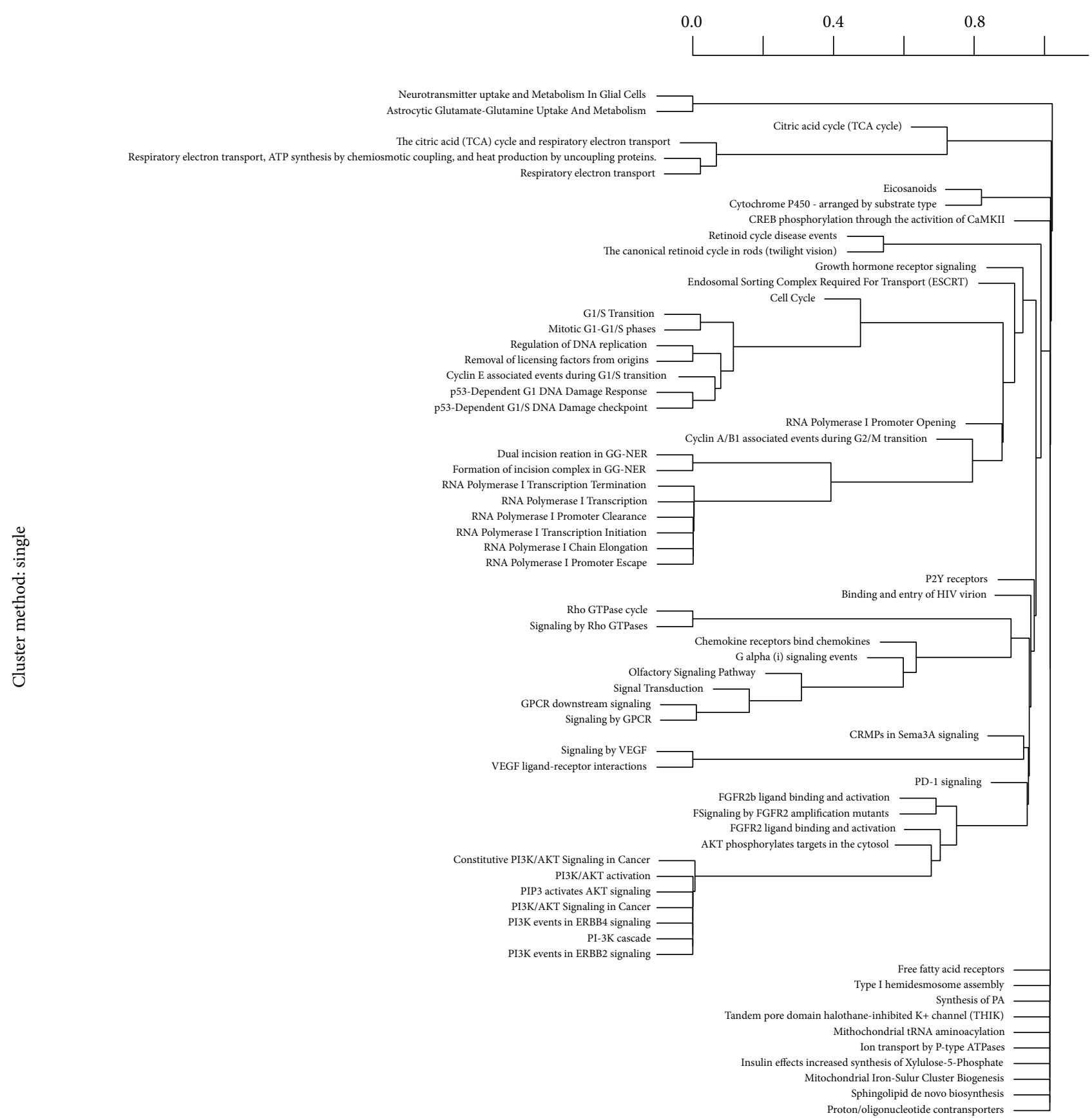

FIGURE 2: Gene content clustering of significant pathways in ALIGATOR analysis under three thresholds for nominal significance.

2556 total genes $(23.3 \%$ and $12.4 \%$, resp.). Using the Gene Ontology (GO) database, over $10 \%$ of genes were assigned to the GO biological processes (GO-BP): "Cell surface receptor linked signal transduction," "G-protein coupled receptor signalling pathway," and "Sensory perception of smell." This final process shows a large genic overlap with the "Signalling by GPCR" Reactome pathway (Figure 2). The "Plasma membrane" (GO cellular component (GO$\mathrm{CC})$ ) and "Olfactory receptor activity" GO molecular function (Go-MF) are also enriched. The full list of significant pathways, with gene count and Benjamini-Hochberg tests, can be found in Supplementary File 1.

\section{Discussion}

In this study, we have identified two candidate regions of genetic association with anti-Ct serological status $\left(p_{\text {EMMAX }}<5 \times 10^{-6}\right)$ in Dutch women attending sexual 
health clinics for which there was at least one supporting SNP in $\mathrm{LD}$ with $R^{2}>0.6$. One of these genomic regions of association was within the protein kinase, CGMP-dependent, type I gene (PRKG1) whilst another was within NPSR1 antisense RNA 1 (NPSR1-AS1), which presumably has a role in posttranscriptional control of the nearby gene neuropeptide $S$ receptor 1 (NPSR1).

The PRKG1 protein is a cytosolic cyclic GMP-dependent protein kinase that has a role in signalling via the cRafMEK1/2-Erk-cMyc/cJUN axis. This may be important in chlamydial disease as the trachoma GWAS [14] identified an association between pathways of signalling through fibroblast growth factor receptors (FGFRs). We suggested that this system could be a crucial component in predisposition to trachomatous scarring. One pathway of signalling from FGFRs to the nucleus involves signal transduction through FRS2, Grb2/SOS, and Ras, which then intersects with the cRafMEK1/2-Erk-cMyc/cJUN axis.

NPSR1 is a G protein-coupled receptor that has been linked to asthma susceptibility [32] and inflammatory bowel disease [33]. NPSR1 interacts with phospholipase C and like both PRKG1 and PREX2 (the best candidate associated gene from the trachoma GWAS [14]) is part of the G proteinmediated cascade of intracellular signalling that centres around the PI3K/Akt pathway of cell cycle regulation. Kechagia and colleagues showed that trachoma fibroblasts could promote Akt phosphorylation in macrophages in an IL-6dependent manner [34]. They went on to suggest that increased IL-6 secretion in trachoma may sustain macrophage activation and survival, with the knock-on effect that this could enhance the contractile activity of the fibroblasts and lead to more severe scarring. Whether the role for the PI3K/Akt pathway in trachoma is to protect against primary infection, or against progression to scarring, or both things, is a matter that requires further study.

This study has some significant limitations. The sample used in this study was very small, and the SNP-based analyses presented in this paper are therefore critically underpowered. Whilst we are confident that the positive signals from the PRKG1 and NPSR1-AS1 region are interesting enough to warrant further study, we recognize that the false-negative rate is very high. To leverage additional power from the small sample, we focussed on pathway-wide analysis that was similar to the one used previously [14]. We determined that the ALIGATOR method was somewhat unstable with regards to the specific pathways that were highlighted at different thresholds of nominal significance (Supplementary Table 2). This is no doubt because more permissive nominal cut-off values (i.e., $p<0.01$ ) lead to a more sensitive analysis that identifies more pathways but lacks the more granular specificity of more conservative thresholds (i.e., $p<0.0001$ ). Collating the results of three different runs of the ALIGATOR analysis allowed us to average across the various sources of error (false negatives versus false positives) whilst identifying pathways. When we assessed the data with functional analysis using DAVID [28], the results pointed towards the importance of cell cycle and $G$ protein-coupled receptor-mediated signalling (Supplementary File 1). By testing for a limited number of pathways whilst utilizing permutation based estimators of significance, we have been able to robustly detect signals of pathway-wide association with chlamydial seropositivity that are almost identical to the findings of the previous study in trachoma.

We identified potential roles for pathways relating to PI3K/Akt signalling, FGFRs, and control of the cell cycle. G-PCRs and their signalling pathways were previously highlighted in trachoma cases $[14,35]$ and also in Chlamydia muridarum infection in the BXD recombinant inbred mouse [36]. Neural growth factor receptor (NGFR) pathways were also strongly associated with serostatus in the Dutch population. Derrick and colleagues [37] previously showed that numerous gene and pathway targets of trachoma-associated microRNA moieties were neuronal related.

We also detected a significant role for olfactory receptor signalling cascades in this study (Supplementary File 1). This could be accounted for by the substantial overlaps between the genes involved in olfactory signalling and the $G$ protein/PI3K signalling system. The olfactory system has however been independently linked to C. pneumoniae infections and subsequent fibrillogenic Alzheimer's like plaque formation in the olfactory centres of mouse brains [38]. Given that such plaques involve collagen restructuring and might be considered akin to the scars that succeed human Ct infections, it seems reasonable to infer that the various Chlamydiaceae can utilise a variety of different, but related, cell surface receptors to gain access to the host cell at different body sites. These diverse receptors may well share a common downstream signalling process via $G$ proteins and particularly via the PI3K pathway. Collectively, these data appear to confirm that $G$ protein-coupled receptor signalling pathways and the cell cycle are associated with chlamydial infections in a way that transcends tissue tropisms and which might be utilised by many or all chlamydial species in their animal hosts. In the absence of an appropriate replication panel for the more granular analyses of the SNP-based association tests, we infer more meaning from the functional overlap of the pathways identified in this study and the previous GWAS in trachoma. We interpret our data in the broadest sense, which is that the key pathways identified between the studies have substantial functional and gene content overlap. A cross-study comparison of the ' $p$ values' for any specific pathway is not however an appropriate way to interpret this overlap, largely because the high false negative risk leads to a certain instability in the association test results. With this small study, we are unfortunately not able to quantify the convergence of the results between the studies.

Another limitation of the study that we highlight is that trait of sero-positivity was measured using a single antigen (MOMP) test. It was not possible to prove unequivocally that all the women who were included in the study as controls had been exposed to $C$. trachomatis infections, nor that coinfection with other microorganisms was not a complicating factor in the clinical presentation of our cases, but this study was carried out in the context of a population of women seeking test and treatment, who were thought to be at very high 
risk for exposure to C. trachomatis STI. Our control group had a uniform antibody titre of $1: 1$, whilst the majority of positives had high titres (mode $1: 200$ ).

We have selected statistical analysis methods for the pathway analysis that allow for a high multiplicity of testing, whilst not imposing the same burden of signal loss that are associated with more rudimentary corrections such as Bonferroni's method. They also collapse highly dimensional data, leading to a better powered 'burden' test. In the light of the robust permutation-based analysis, we used the remarkable similarities between our findings and those of the previous trachoma GWAS; it is extremely unlikely that these associations have been identified in both the trachoma and STI tropisms either by chance or because of systematic errors in our analysis approach.

By focussing on a trait that is generally accepted to indicate lifetime responses to infectious challenge, we believe that we have identified pathway-wide genotypes that appear to associate with protection from infection in some women. This protection could take the form of a partial or complete refractoriness to primary cellular infection, or a rapid, intense, and highly effective innate immune response.

\section{Conclusions}

Between the tissue tropisms of the eye and the genital tract, the pathway-wide genetic risk and protection patterns overlap to a substantial degree. This supports a model under which common pathways of intracellular signalling might be utilised by all Chlamydiaceae, even though the specific receptors that mediate introgression into the cell might be highly variable.

\section{Conflicts of Interest}

The authors declare no competing interests.

\section{Authors' Contributions}

Servaas A. Morré, Sander Ouburg, Martin J. Holland, and Chrissy H. Roberts designed the study. Sander Ouburg, Servaas A. Morré, and Henry J.C. de Vries performed the clinical collections and laboratory tests. Mark D. Preston, Martin J. Holland, and Chrissy H. Roberts performed the computational work and statistical analyses. All authors wrote, reviewed, and approved the manuscript. Chrissy $\mathrm{H}$. Roberts and Sander Ouburg contributed equally to the work.

\section{Acknowledgments}

The authors are very grateful to the participants of this study. This work was supported in part by the European EuroTransBio Grant (reference no. 110012 ETB) and the Eurostars TubaTEST grant (reference number E!9372). Chrissy H. Roberts was supported by the Wellcome Trust Institutional Strategic Support Fund (105609/Z/14/Z).

\section{Supplementary Materials}

Supplementary 1. Results of pathway enrichment analysis using Database for Annotation, Visualization and Integrated Discovery (DAVID).

Supplementary 2. Supplementary Figure 1: results of GWAS analysis using EMMAX|QQ plot. Supplementary Table 1: complete list of SNPs with pEMMAX $<1 \times 10-6$. Supplementary Table 2: permuted $\mathrm{p}$ values for Reactome pathways with significant enrichment in chlamydial seropositivity: ALIGATOR analysis.

\section{References}

[1] J. Rowley, I. Toskin, and F. Ndowa, Global Incidence and Prevalence of Selected Curable Sexually Transmitted Infections, 2008, WHO Press, World Health Organization, Geneva, Switzerland, 2008.

[2] V. H. Hu, M. J. Holland, and M. J. Burton, "Trachoma: protective and pathogenic ocular immune responses to Chlamydia trachomatis," PLoS Neglected Tropical Diseases, vol. 7, no. 2, article e2020, 2013.

[3] M. Guzey, I. Ozardali, E. Basar, G. Aslan, A. Satici, and S. Karadede, "A survey of trachoma: the histopathology and the mechanism of progressive cicatrization of eyelid tissues," Ophthalmologica, vol. 214, no. 4, pp. 277-284, 2000.

[4] A. A. al-Rajhi, A. Hidayat, A. Nasr, and M. al-Faran, "The histopathology and the mechanism of entropion in patients with trachoma," Ophthalmology, vol. 100, no. 9, pp. 1293-1296, 1993.

[5] T. Darville and T. J. Hiltke, "Pathogenesis of genital tract disease due to Chlamydia trachomatis," The Journal of Infectious Diseases, vol. 201, no. S2, Supplement 2, pp. 114-S125, 2010.

[6] C. L. Haggerty, S. L. Gottlieb, B. D. Taylor, N. Low, F. Xu, and R. B. Ness, "Risk of sequelae after Chlamydia trachomatis genital infection in women," The Journal of Infectious Diseases, vol. 201, no. S2, Supplement 2, pp. 134-S155, 2010.

[7] S. Resnikoff, D. Pascolini, D. Etya'ale et al., "Policy and Practice Global data on visual impairment in the year 2002," Bulletin of the World Health Organization, vol. 82, no. 11, pp. 844-851, 2004.

[8] P. J. Dolin, H. Faal, G. J. Johnson, J. Ajewole, A. A. Mohamed, and P. S. Lee, "Trachoma in the Gambia," British Journal of Ophthalmology, vol. 82, no. 8, pp. 930-933, 1998.

[9] S. P. Mariotti, D. Pascolini, and J. Rose-Nussbaumer, "Trachoma: global magnitude of a preventable cause of blindness," British Journal of Ophthalmology, vol. 93, no. 5, pp. 563-568, 2009.

[10] I. G. M. van Valkengoed, S. A. Morré, A. J. C. van den Brule, C. J. Meijer, L. M. Bouter, and A. J. Boeke, "Overestimation of complication rates in evaluations of Chlamydia trachomatis screening programmes-implications for cost-effectiveness analyses," International Journal of Epidemiology, vol. 33, no. 2, pp. 416-425, 2004.

[11] S. A. Morre, C. Munk, K. Persson et al., "Comparison of three commercially available peptide-based immunoglobulin G (IgG) and IgA assays to microimmunofluorescence assay for detection of Chlamydia trachomatis antibodies," Journal of Clinical Microbiology, vol. 40, no. 2, pp. 584-587, 2002.

[12] P. Holmans, E. K. Green, J. S. Pahwa et al., "Gene ontology analysis of GWA study data sets provides insights into the 
biology of bipolar disorder," The American Journal of Human Genetics, vol. 85, no. 1, pp. 13-24, 2009.

[13] P. C. Sham and S. M. Purcell, "Statistical power and significance testing in large-scale genetic studies," Nature Reviews Genetics, vol. 15, no. 5, pp. 335-346, 2014.

[14] C. h. Roberts, C. S. Franklin, P. Makalo et al., "Conjunctival fibrosis and the innate barriers to Chlamydia trachomatis intracellular infection: a genome wide association study," Scientific Reports, vol. 5, no. 1, article 17447, 2015.

[15] Q.-R. Chen, R. Braun, Y. Hu et al., "Multi-SNP analysis of GWAS data identifies pathways associated with nonalcoholic fatty liver disease," PLoS One, vol. 8, no. 7, article e65982, 2013.

[16] R. Braun and K. Buetow, "Pathways of distinction analysis: a new technique for multi-SNP analysis of GWAS data," PLoS Genetics, vol. 7, no. 6, article e1002101, 2011.

[17] M. C. Wu, S. Lee, T. Cai, Y. Li, M. Boehnke, and X. Lin, "Rarevariant association testing for sequencing data with the sequence kernel association test," The American Journal of Human Genetics, vol. 89, no. 1, pp. 82-93, 2011.

[18] L. Matthews, G. Gopinath, M. Gillespie et al., "Reactome knowledgebase of human biological pathways and processes," Nucleic Acids Research, vol. 37, Supplement 1, pp. D619D622, 2009.

[19] Y. Y. Teo, M. Inouye, K. S. Small et al., "A genotype calling algorithm for the Illumina BeadArray platform," Bioinformatics, vol. 23, no. 20, pp. 2741-2746, 2007.

[20] J. H. Zhao, "Gap: genetic analysis package," Journal of Statistical Software, vol. 23, no. 8, 2007.

[21] B. Howie, C. Fuchsberger, M. Stephens, J. Marchini, and G. R. Abecasis, "Fast and accurate genotype imputation in genomewide association studies through pre-phasing," Nature Genetics, vol. 44, no. 8, pp. 955-959, 2012.

[22] B. N. Howie, P. Donnelly, and J. Marchini, "A flexible and accurate genotype imputation method for the next generation of genome-wide association studies," PLoS Genetics, vol. 5, no. 6, article e1000529, 2009.

[23] O. Delaneau, J. Marchini, and J.-F. Zagury, "A linear complexity phasing method for thousands of genomes," Nature Methods, vol. 9, no. 2, pp. 179-181, 2012.

[24] J. O'Connell, D. Gurdasani, O. Delaneau et al., "A general approach for haplotype phasing across the full Spectrum of relatedness," PLoS Genetics, vol. 10, no. 4, article e1004234, 2014.

[25] L. Southam, K. Panoutsopoulou, N. W. Rayner et al., "The effect of genome-wide association scan quality control on imputation outcome for common variants," European Journal of Human Genetics, vol. 19, no. 5, pp. 610-614, 2011.

[26] G. R. Abecasis, A. Auton, L. D. Brooks et al., "An integrated map of genetic variation from 1,092 human genomes," Nature, vol. 491, no. 7422, pp. 56-65, 2012.

[27] H. M. Kang, J. H. Sul, S. K. Service et al., "Variance component model to account for sample structure in genome-wide association studies," Nature Genetics, vol. 42, no. 4, pp. 348-354, 2010.

[28] D. W. Huang, B. T. Sherman, and R. A. Lempicki, "Systematic and integrative analysis of large gene lists using DAVID bioinformatics resources," Nature Protocols, vol. 4, no. 1, pp. 44-57, 2009.

[29] G. S. Wills, P. J. Horner, R. Reynolds et al., "Pgp3 antibody enzyme-linked immunosorbent assay, a sensitive and specific assay for seroepidemiological analysis of Chlamydia trachomatis infection," Clinical and Vaccine Immunology, vol. 16, no. 6, pp. 835-843, 2009.

[30] N. K. Björkström, P. Riese, F. Heuts et al., "Expression patterns of NKG2A, KIR, and CD57 define a process of CD56 dim NKcell differentiation uncoupled from NK-cell education," Blood, vol. 116, no. 19, pp. 3853-3864, 2010.

[31] S. Ouburg, J. Spaargaren, J. E. den Hartog et al., "The CD14 functional gene polymorphism $-260 \mathrm{C}>\mathrm{T}$ is not involved in either the susceptibility to Chlamydia trachomatis infection or the development of tubal pathology," BMC Infectious Diseases, vol. 5, no. 1, article 114, 2005.

[32] T. Laitinen, A. Polvi, P. Rydman et al., "Characterization of a common susceptibility locus for asthma-related traits," Science, vol. 304, no. 5668, pp. 300-304, 2004.

[33] M. Henström, M. Zucchelli, C. Söderhäll et al., "NPSR1 polymorphisms influence recurrent abdominal pain in children: a population-based study," Neurogastroenterology \& Motility, vol. 26, no. 10, pp. 1417-1425, 2014.

[34] J. Z. Kechagia, D. G. Ezra, M. J. Burton, and M. Bailly, "Fibroblasts profiling in scarring trachoma identifies IL-6 as a functional component of a fibroblast-macrophage profibrotic and pro-inflammatory feedback loop," Scientific Reports, vol. 6, no. 1, article 28261, 2016.

[35] M. J. Burton, S. N. Rajak, J. Bauer et al., "Conjunctival transcriptome in scarring trachoma," Infection and Immunity, vol. 79, no. 1, pp. 499-511, 2011.

[36] Y. Su, X. Wang, E. Jones et al., "Host genetics and upper genital tract disease in Chlamydia muridarum infected mice: a forward genetic approach with translational implications," in Proceedings of the 13th International Symposium on Human Chlamydial Infections, pp. 261-264, Pacific Grove, CA, USA, 2014.

[37] T. Derrick, C. Roberts, M. Rajasekhar et al., "Conjunctival microRNA expression in inflammatory trachomatous scarring," PLoS Neglected Tropical Diseases, vol. 7, no. 3, article e2117, 2013.

[38] C. S. Little, C. J. Hammond, A. MacIntyre, B. J. Balin, and D. M. Appelt, "Chlamydia pneumoniae induces Alzheimerlike amyloid plaques in brains of BALB/c mice," Neurobiology of Aging, vol. 25, no. 4, pp. 419-429, 2004. 


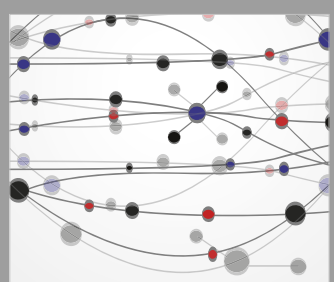

The Scientific World Journal
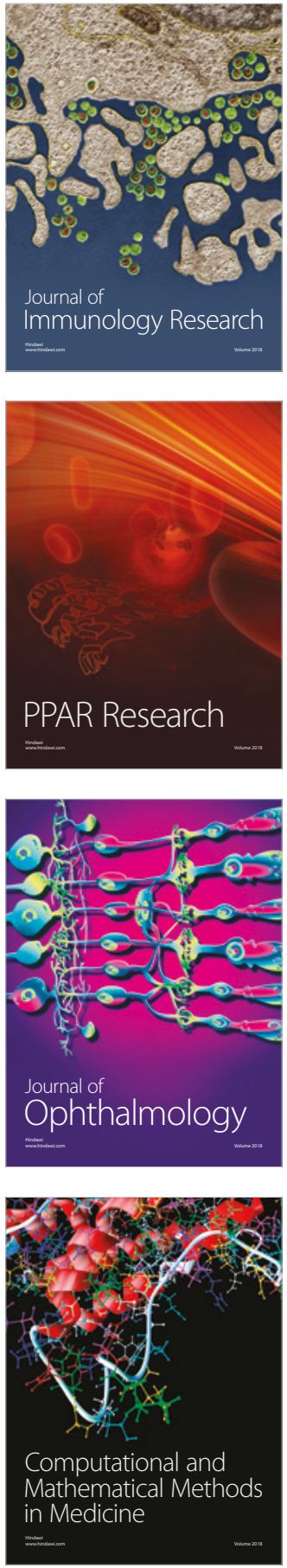

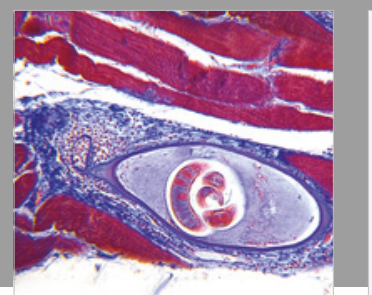

Gastroenterology Research and Practice

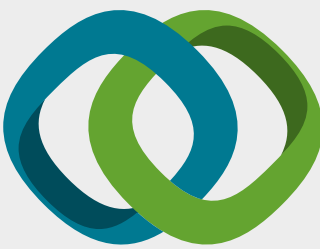

\section{Hindawi}

Submit your manuscripts at

www.hindawi.com
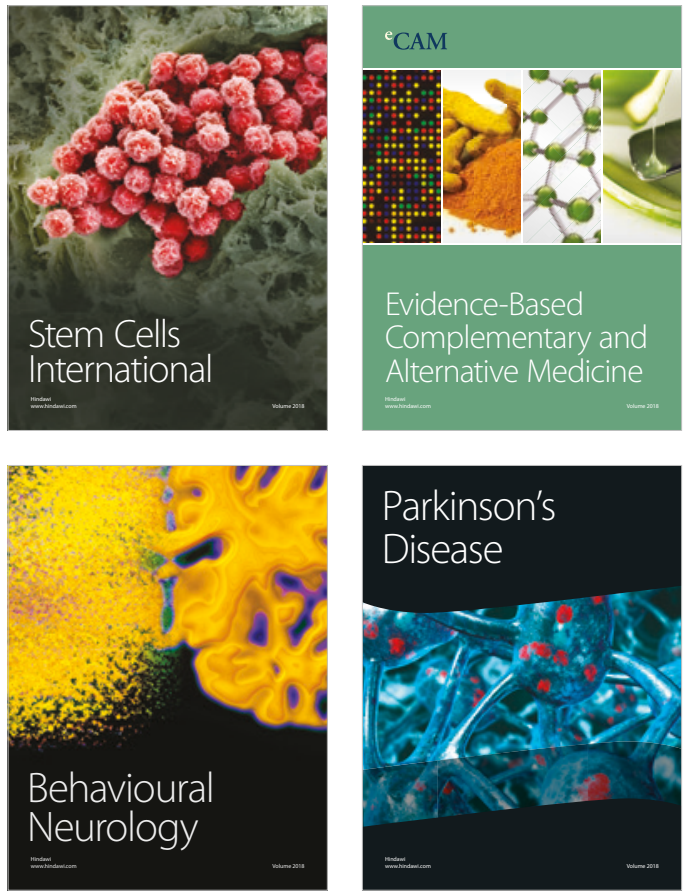

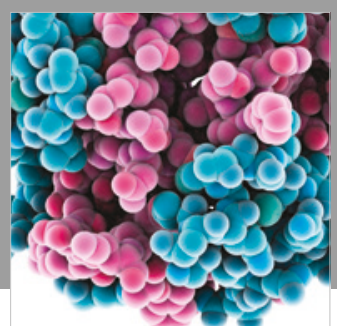

ournal of

Diabetes Research

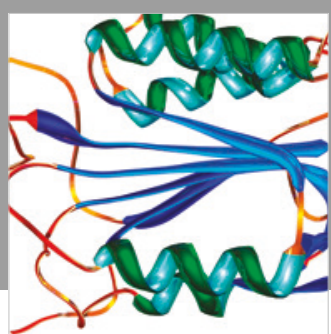

Disease Markers
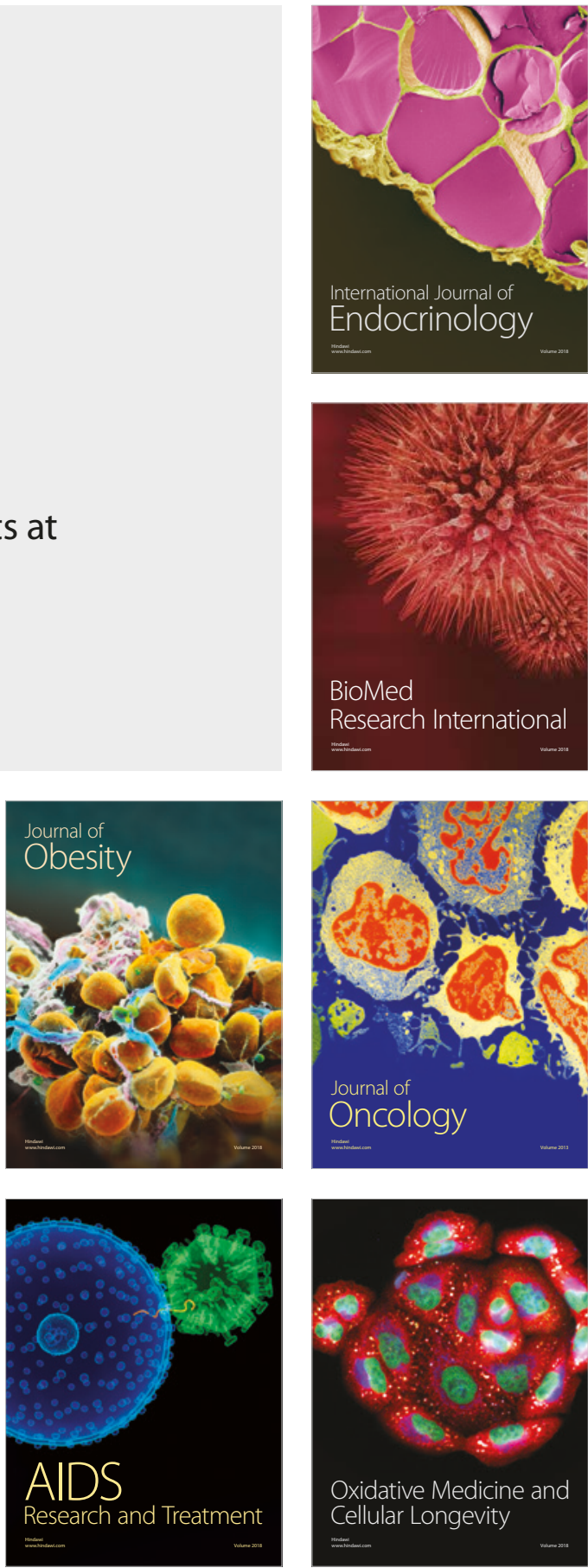